\title{
Electromagnetically driven, fast opening and closing gas jet valve
}

\author{
Mahadevan Krishnan and Kristi Wilson Elliott \\ Alameda Applied Sciences Corporation, San Leandro, California 94577, USA \\ C. G. R. Geddes, R. A. van Mourik, and W. P. Leemans \\ Lawrence Berkeley National Laboratory, Berkeley, California 94720, USA \\ H. Murphy and M. Clover \\ Science Applications International Corporation, La Jolla, California 92121, USA
}

(Received 16 July 2010; published 10 March 2011)

\begin{abstract}
The design and performance are presented of an electromagnetically driven gas valve [M. Krishnan, J. Wright, and T. Ma, Proceedings of the 13th Advanced Accelerator Concepts Workshop, Santa Cruz, CA, AIP Conf. Proc. No. 1086 (AIP, New York, 2008)] that opens in $<100 \mu$ s, closes in $<500 \mu$ s, and can operate at pressures of $\sim 1000$ psia to drive supersonic nozzles. Such a valve has applications to laserplasma accelerators, where the fast opening and closing would allow sharper edges to the flow and also allow higher rep-rate operation without loading the vacuum chamber. The valve action is effected by a flyer plate accelerated by the electromagnetic impulse of a low inductance, spiral wound, strip-line coil driven by a capacitor. Gas flows out of the valve when the seal between this flyer plate and the valve seat is broken. The electromagnetic force greatly exceeds the restoring forces provided by a spring and the gas pressure against the valve seat. Piezoresistive sensor and laser interferometer measurements of flow show that the valve opens in $\sim 100 \mu \mathrm{s}$ for all pressures up to $800 \mathrm{psia}$. The closing time is $500 \mu \mathrm{s}$, set by the spring constant and mass. The prototype valve has been operated with helium at $0.5 \mathrm{~Hz}$ and at $500 \mathrm{psia}$ for $\sim 1$ hour at a time with no cooling.
\end{abstract}

DOI: 10.1103/PhysRevSTAB.14.033502

PACS numbers: 47.40.Ki, 41.75.Jv

\section{INTRODUCTION}

There are numerous fields in which fast pulses of supersonic gas jets are useful. In free molecular beams, the challenge is to produce intense, high Mach number beams while maintaining a low base vacuum pressure to conduct precise measurements of collision or ionization cross sections. In pulsed x-ray machines, a supersonic shell or cylinder of gas is compressed by high currents to form a dense, hot plasma pinch, which radiates the desired $\mathrm{x}$ rays in a short pulse. The emerging field of laser-plasma acceleration (LPA) has demonstrated electron accelerators with unprecedented electric field gradients [1]. Supersonic, highly collimated gas jets and gas-filled capillary discharge waveguides are two primary targets of choice for LPA. Present gas jets have lengths of only $2-4 \mathrm{~mm}$ at densities of $1-4 \times 10^{19} \mathrm{~cm}^{-3}$, sufficient for self-trapping and electron acceleration to energies up to $\sim 150 \mathrm{MeV}$ [1-3]. Our fast valve can drive nozzles with larger throats and, hence, produce $\sim 1 \mathrm{~cm}$ gas jets at high density, allowing for $\sim 1 \mathrm{GeV}$ acceleration. Capillary structures $3 \mathrm{~cm}$ long have been used to accelerate beams up to $1 \mathrm{GeV}$ [4]. Gas

Published by American Physical Society under the terms of the Creative Commons Attribution 3.0 License. Further distribution of this work must maintain attribution to the author(s) and the published article's title, journal citation, and DOI. jets with uniform or controlled density profiles may be used to control injection [5], and are being integrated into capillary experiments to add tuning of density [6]. A third application is in conventional accelerators, where a metered pulse of gas into a beam line or test cell is injected to, for example, control particle beam stripping or to carry out nuclear spectroscopy measurements.

Supersonic jets are used because they provide short "ramp" lengths at the sides of the jet where the density increases from zero to a peak value, and a uniform flattop density region in the center. This requires high pressure operation, and increasing valve operating pressure allows, for a given gas density needed by the application, operation at higher Mach number for reduced edge ramp length. In LPAs, this can be important to optimize coupling of the laser, or to create density gradients for injection [5].

A fast opening and closing gas valve is essential to applications. For example, in LPAs, to minimize distortion of the drive laser pulse on its way to the focus, the background pressure must be kept sufficiently low ( $<1$ mtorr). Typical commercially available gas valves that require opening and closing times of several milliseconds in duration can then severely limit the repetition rate of the device. Fast opening times can minimize pump loading with high repetition rates and potentially increase control over gas profile by minimizing disturbance before laser arrival, when integrated in capillaries. In conventional 
accelerators, the challenge is to control the mass injected with sufficient precision so that only the region of interest is filled with gas, while the rest of the accelerator structure can remain at high vacuum; in other words, the gas burst must be fast enough so that a high vacuum event may occur before the injected gas reaches the high vacuum region to perturb it.

Prior designs of similar fast electromagnetic valves have demonstrated fast opening and closing as well as high repetition rate operation, but at lower operating pressures than those optimal for many applications. Pioneering work by Gentry and Giese [7] demonstrated an elegant, tuning fork valve mechanism that produced $\sim 10 \mu$ s pulses of $\mathrm{He}$ molecular beams, with water cooling of the valve body. Corat et al. [8] later demonstrated a different electromagnetic actuator in which a static coil repels a metallic flyer plate. This combination was shown to operate at up to $100 \mathrm{~Hz}$ with $\sim 100 \mu$ s pulses at nitrogen pressure up to 300 psia. Adams et al. [9] described a similar design that operated at up to $35 \mathrm{~Hz}$ and at pressures of $\sim 15$ psia.

Here we present the design and performance of a novel gas valve that has the fast opening and closing attributes of earlier valves, but is capable of operation at $\sim 1500$ psia pressures benefiting applications such as LPAs. The first section describes the design of the valve, which relies on a fast rise time electromagnetic driver, enabling submillisecond operating times and high backing pressures [10]. Section II describes design of the gas nozzles used with the valve. Measurements of the output flow are presented in Sec. III, showing that the valve opens in $<100 \mu \mathrm{s}$ and closes in $\sim 500 \mu \mathrm{s}$. These measurements are confirmed using gas interferometry in Sec. IV. Thermal issues for operation of the valve at $1 \mathrm{~Hz}$ and higher repetition rates for long duration times are discussed in Sec. V, followed by conclusions.

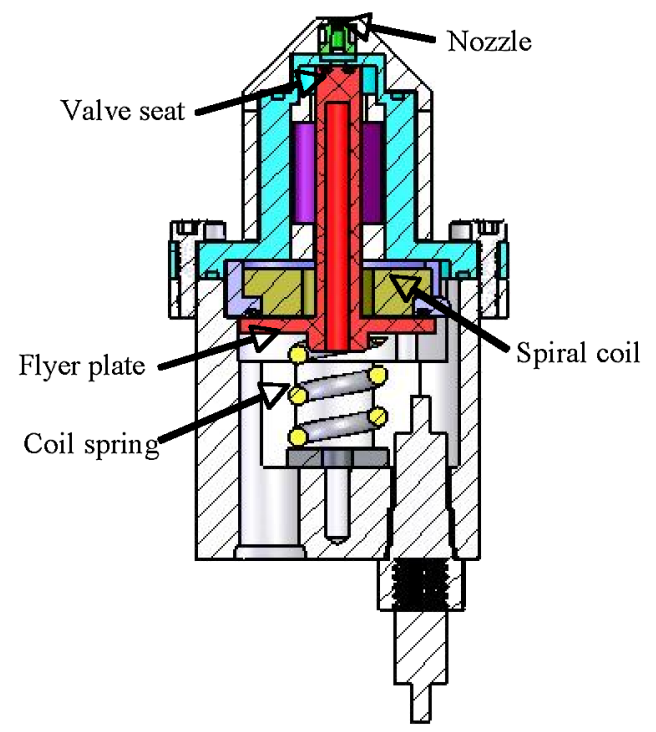

FIG. 1. Cross-section view of Alameda Applied Sciences Corporation's ultrafast gas valve.

\section{DESIGN OF FLYER-PLATE DRIVEN FAST GAS JET VALVE}

The valve described in this paper operates by driving a fast $(\sim 100 \mu \mathrm{s})$, high current $(\sim 1 \mathrm{kA})$ pulse into an electromagnetic coil. An aluminum flyer plate is pressed against this coil and is repelled by the eddy current induced in it. The flyer plate is backed by a stiff spring. The natural harmonic half period of the flyer-plate/spring system is $\sim 600 \mu \mathrm{s}$. The electromagnetic force acts on this oscillator for $\sim 100 \mu \mathrm{s}$. Thus the motion of the flyer plate is akin to a pendulum that is excited by a sharp impulse. The plate moves back $\sim 1 \mathrm{~mm}$ in $\sim 100 \mu \mathrm{s}$, then returns to its seat in $\sim 600 \mu \mathrm{s}$. The flyer plate has a long stub that seals against the valve face, to which a nozzle is attached. This stub minimizes the volume between the valve seat and the nozzle throat, which allows fast rise time of the gas from the nozzle.

Figures 1 and 2 show a cross-section view and photograph of the fast valve. Figure 1 shows the Al flyer plate backed by a $205 \mathrm{~N} / \mathrm{mm}$ compression spring and pressed up against the spiral wound coil. The $25 \mathrm{~mm}$ diameter coil

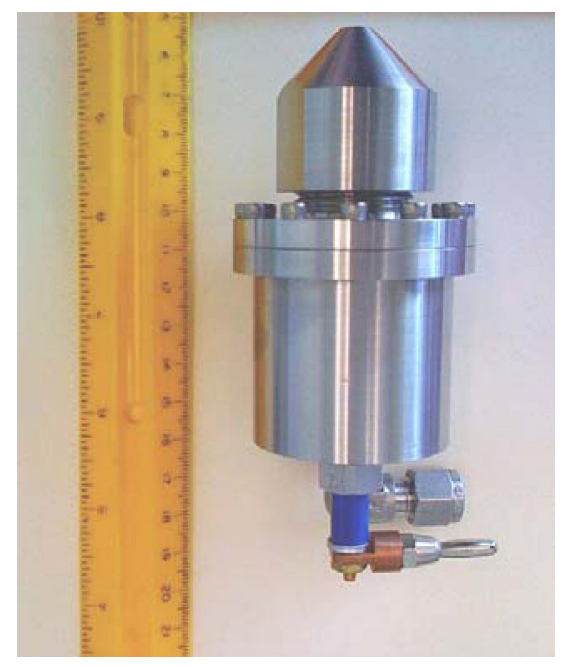

FIG. 2. Photograph of assembled valve.

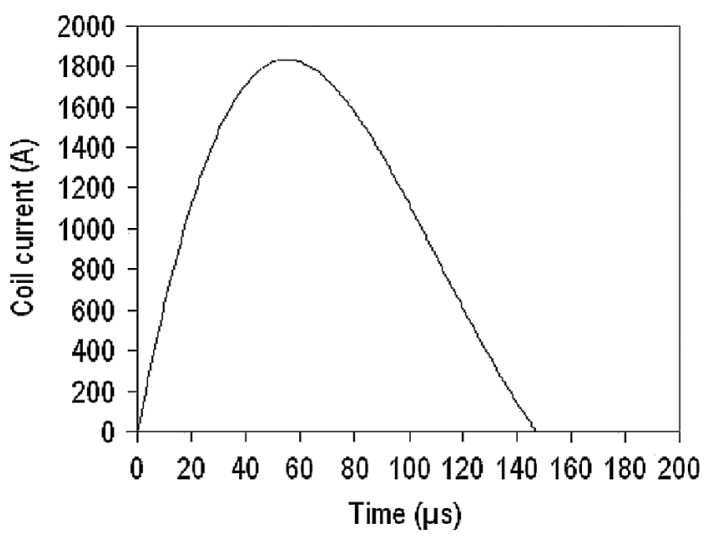

FIG. 3. Current to spiral coil. 


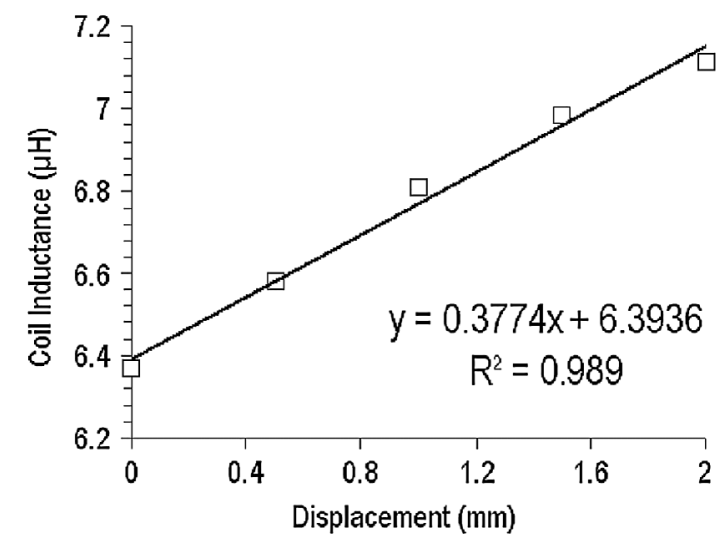

FIG. 4. Coil inductance measurement.

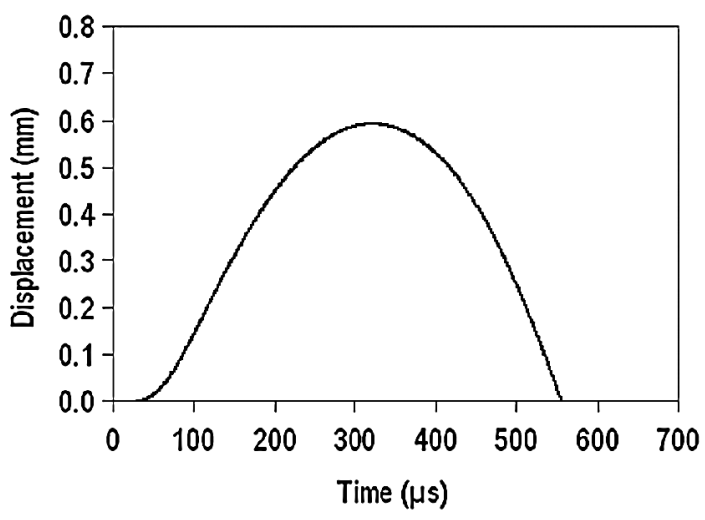

FIG. 5. Calculated motion of flyer plate.

is wound from $3 \mathrm{~mm}$ wide $\mathrm{Cu}$ strip and insulated by a thin Kapton strip. The DC resistance of the $\approx 22$ turn coil is $0.04 \Omega$. The flyer plate has a long hollow snout that seals against a $4 \mathrm{~mm}$ diameter O-ring close to the base of the nozzle bobbin. The snout is made hollow to minimize the flyer-plate mass. At a typical charge voltage of $350 \mathrm{~V}$, a current of $\sim 2 \mathrm{kA}$ with a $\sim 140 \mu \mathrm{s}$ half period in the $\sim 6 \mu \mathrm{H}$ coil imparts a $\sim 0.07$ Ns impulse to the $\sim 9 \mathrm{~g} \mathrm{Al}$ flyer plate.

Figure 3 shows the current to the spiral coil from the $370 \mu \mathrm{F} / 350 \mathrm{~V}$ capacitor bank. The current reaches a peak of $1835 \mathrm{~A}$ in $\approx 60 \mu \mathrm{s}$ and has a half period of $140 \mu \mathrm{s}$. Figure 4 plots the measured coil inductance vs displacement from the metal base. The linear fit shown in the inset gives the slope $d L / d x$, which is proportional to the force on the flyer plate, i.e.,

$$
F_{m}=0.5 I^{2}(d L / d x) .
$$

The mass of the flyer plate, the spring stiffness, and Eq. (1) allow the motion of the flyer plate to be calculated.

The calculated motion of the flyer plate is shown in Fig. 5. This shows that the flyer plate begins to move at the peak in current and reaches a maximum excursion of $0.55 \mathrm{~mm}$ in $350 \mu \mathrm{s}$, after which it returns to the valve seat in $\sim 550 \mu$ s after the start of current.

\section{GAS JET NOZZLE DESIGN}

For an LPA (or other) application, the valve must be coupled with a suitable supersonic nozzle to create the well-defined gas jet target. We have designed two types of supersonic Laval nozzles to be coupled to the valve described above to produce suitable targets for LPAs. The first nozzle is a so-called method of characteristics design, in which the hyperbolic equations are solved to give a shock-free contour everywhere along the supersonic expansion, and a so-called straight nozzle, in which the supersonic section is a simple conical expansion from the throat to the exit. Since the straight nozzle is easier to manufacture, it is also relevant to ask whether there are significant differences in the flows that exit these two types of nozzles.

We performed numerical simulations of three different candidate nozzle shapes. The geometric specifications of the three nozzles are listed in Table I. All nozzle profiles are axisymmetric about the $z$ axis in the $r, z$ coordinate system and represent converging diverging nozzles, so that they all can be considered to be Laval nozzles. The diverging part of the $4.6 \mathrm{~mm}$ straight nozzle profile is a straight contour from the $0.29 \mathrm{~mm}$ radius nozzle throat to the $1.1 \mathrm{~mm}$ radius nozzle exit with a $10^{\circ}$ slant angle. The length of the diverging section is $4.65 \mathrm{~mm}$ such that its slant angle is matched to the Mach angle 1/M [11,12]. This nozzle has been in use at LOASIS/LBNL. The second straight nozzle also has a contour that is a simple cone, whose projection on a plane is a straight line with an approximately $6^{\circ}$ slant angle, with an expansion length of $8 \mathrm{~mm}$. The third nozzle's contour has been determined

TABLE I. Geometric specifications for the various nozzle configurations.

\begin{tabular}{lcccc}
\hline \hline Nozzle name & Throat radius $(\mathrm{mm})$ & Exit radius $(\mathrm{mm})$ & Length $(\mathrm{mm})$ & \multicolumn{1}{c}{ Description } \\
\hline $4.6 \mathrm{~mm}$ straight nozzle & 0.29 & 1.1 & 4.65 & $\begin{array}{l}\text { Straight wall contour with a } 10^{\circ} \text { slant angle for } \\
\text { diverging section }\end{array}$ \\
$8 \mathrm{~mm}$ straight nozzle & 0.29 & 1.1 & 8.0 & $\begin{array}{l}\text { Straight wall contour with approximately a } 6^{\circ} \\
\text { slant angle for diverging section } \\
\text { Curved wall contour designed using a MOC } \\
\text { code for diverging section }\end{array}$ \\
\hline \hline
\end{tabular}


numerically using the method of characteristics (MOC); this nozzle has the same $8 \mathrm{~mm}$ length as does the second straight nozzle for direct comparison. The purpose of the numerical simulations was to evaluate what the differences are, in the exit flows from the three nozzles. If the differences are not significant from the standpoint of laserplasma acceleration (LPA), that would allow straight nozzles to be used that are less expensive to fabricate.

\section{Simulation results}

The tool used for the flow simulations is the RAGE code, an enhanced version of SAGE (SAIC Adaptive Grid Eulerian). SAGE is a multidimensional (1D, 2D, 3D), multimaterial, massively parallel, Eulerian hydrodynamics code for use in solving the Euler equation in a variety of highdeformation flow problems. It uses unit aspect ratio computational cells in all geometries (1D, 2D, 3D Cartesian, 1D, 2D cylindrical, and 1D spherical). The mesh is refined locally every computational cycle by an adaptive mesh refinement algorithm that looks at local spatial variation of physical quantities (pressure, density, material boundaries, etc.) and then bisects or recombines cells so as to maintain a desired degree of resolution. The RAGE code is built on top of SAGE by adding various radiation packages and is typically used for problems in which radiative transport of energy is important. These added capabilities of RAGE were not needed for the simulations reported here, which are essentially 2D axisymmetric supersonic expansions of an ideal monatomic gas.

The quantities of interest for LPA targets, and for comparison with interferometric diagnostics, are the gas density contours and lineouts, especially those from where the flow exhausts from the nozzle exit up to a few mm away from the exit. In the simulations it was verified that the flow field was at steady state $3 \mathrm{~mm}$ downstream from the nozzle exit before the calculation was stopped. For the $4.6 \mathrm{~mm}$ straight nozzle this was $10 \mu$ s past the time that the 25 atm He was allowed to expand downwards through the nozzle throat, through the diverging conical straight walled section, and out into the vacuum chamber. Figure 6 shows radial density lineouts for the $4.6 \mathrm{~mm}$ straight nozzle at locations $0,1,2$, and $3 \mathrm{~mm}$ downstream of the nozzle exit, and the color inset shows the density contours for that nozzle. For the inset the $z$ axis runs top to bottom and $z$ increases down the axis. The $r$ axis runs from left to right and $r$ increases toward the right. The flow starts from the stagnation chamber at the top and expands downward through the nozzle throat, into the diverging section of the nozzle, and out into the vacuum chamber.

In the plot area of the figure, the black line is the density profile at a $z$ location of the nozzle exit which is $0 \mathrm{~mm}$ downstream from the exit. It shows the density at that location as a function of radius. The next line down (blue dotted) is the density lineout $1 \mathrm{~mm}$ downstream of the exit. Note that this line is lower in value than the black line and

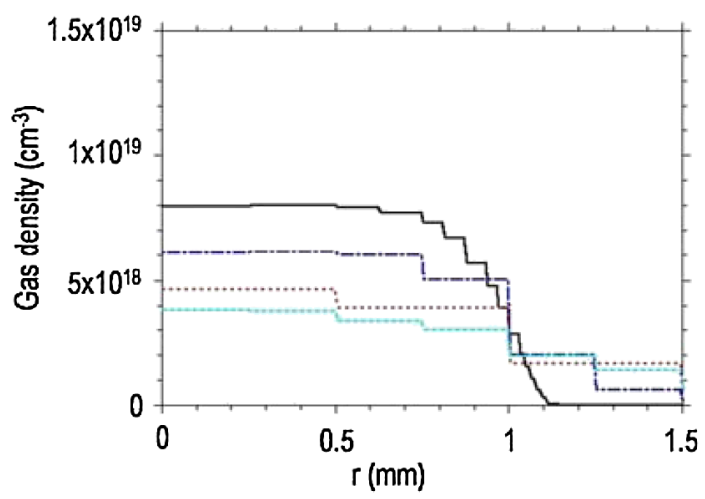

FIG. 6. $4.6 \mathrm{~mm}$ straight nozzle radial density lineouts for 0 (black), 1 (blue), 2 (red), and 3 (teal) mm downstream of the nozzle exit at $10 \mu \mathrm{s}$.

has a tail expanding further out radially to the right. It is beyond the nozzle exit and the gas is turning as it expands into the vacuum chamber. The red dotted line shows the density lineout at $2 \mathrm{~mm}$ downstream from the nozzle exit, while the teal line represents the density $3 \mathrm{~mm}$ past the nozzle exit. Further out into the flow field shows decreasing densities in all directions since the gas is now expanding outside any nozzle walls.

Figures 7 and 8 show radial density lineouts for the $8 \mathrm{~mm}$ straight nozzle and the $8 \mathrm{~mm}$ MOC nozzle, respectively. These calculations were run out to $20 \mu \mathrm{s}$, to ensure that steady-state conditions were evident $3 \mathrm{~mm}$ past the nozzle exits. This was required because the nozzles are longer than the $4.6 \mathrm{~mm}$ straight nozzle.

Figure 9 shows an overlay of the radial density lineouts for each of the three nozzle profiles at a location $3 \mathrm{~mm}$ downstream from each of the nozzle exits. The $4.6 \mathrm{~mm}$ straight nozzle is approximately 2 times lower in density near the $z$ axis when compared to the two $8 \mathrm{~mm}$ nozzles both at $3 \mathrm{~mm}$ and at the exit plane. The $4.6 \mathrm{~mm}$ straight nozzle has a slant angle that is matched to the (design) Mach number of 5.6, whereas the MOC nozzles have a different slant angle, yet there is no strong shock evident in

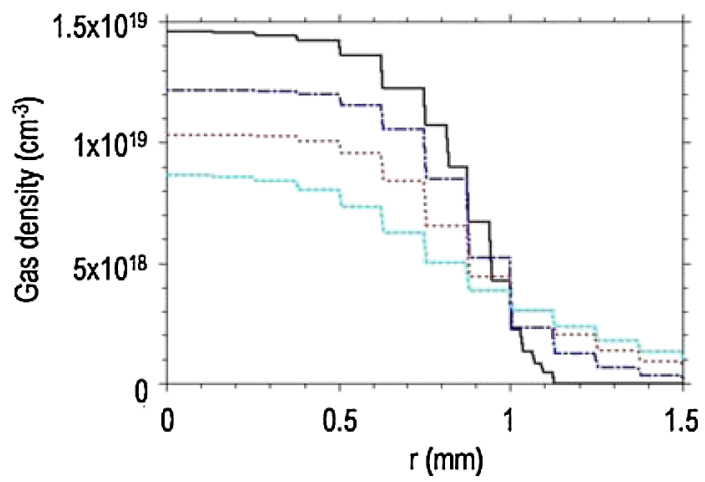

FIG. 7. $8 \mathrm{~mm}$ straight nozzle radial density lineouts for 0 (black), 1 (blue), 2 (red), and 3 (teal) mm downstream of the nozzle exit at $20 \mu \mathrm{s}$. 


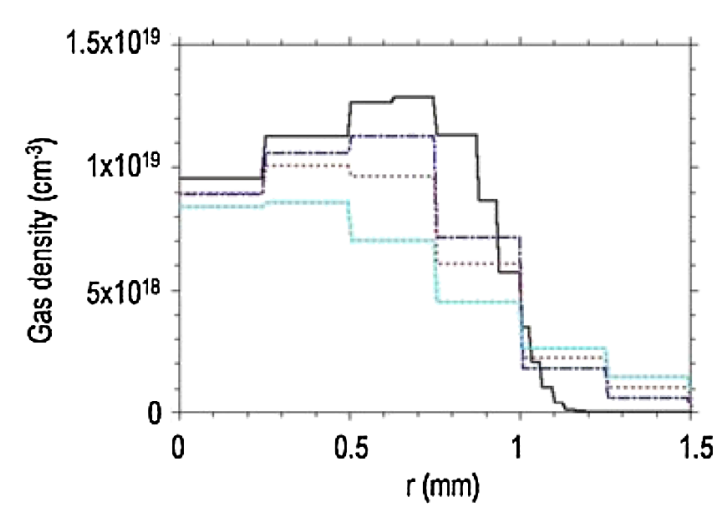

FIG. 8. $8 \mathrm{~mm}$ MOC nozzle radial density lineouts for 0 (black), 1 (blue), 2 (red), and 3 (teal) mm downstream of the nozzle exit at $20 \mu \mathrm{s}$.

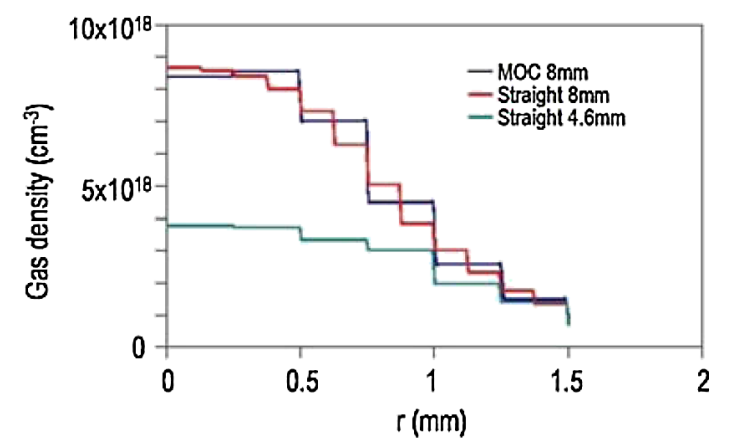

FIG. 9. Radial density lineouts at $3 \mathrm{~mm}$ downstream of the nozzle exit for each of the three nozzles at steady state.

the RAGE simulation that could account for a factor of 2 higher exit density. This is most likely an artifact due to different boundary conditions used between the plenum and throat for the RAGE simulations of the 4.6 and $8 \mathrm{~mm}$ nozzles. If the expansion were ideal, the area ratios of the three nozzles should lead to the same density at exit plane. Additionally, the density lineouts of the $8 \mathrm{~mm}$ MOC nozzle and the $8 \mathrm{~mm}$ straight nozzle almost overlay one another at $3 \mathrm{~mm}$ but the MOC nozzle shows a hollow profile closer to the nozzle (Fig. 8). It is also remarkable that the $3 \mathrm{~mm}$ radial density lineout for the $8 \mathrm{~mm}$ straight nozzle does not visibly spread out more than that for the $8 \mathrm{~mm}$ MOC nozzle.

Figure 10 shows the Mach number contours for the $8 \mathrm{~mm} \mathrm{MOC}$ and the $8 \mathrm{~mm}$ straight nozzles with superimposed flow particle velocity vectors. A careful inspection shows that the flow field exiting the MOC nozzle near the exit is indeed very aligned with the $z$ axis while that for the $8 \mathrm{~mm}$ straight nozzle shows some radial expansion, though not significantly.

The RAGE simulations allow comparison and selection of the appropriate nozzle for applications. Prior work [11] has shown results using a similar simulation code. For an LPA, the primary desire is to have a uniform flattop in density

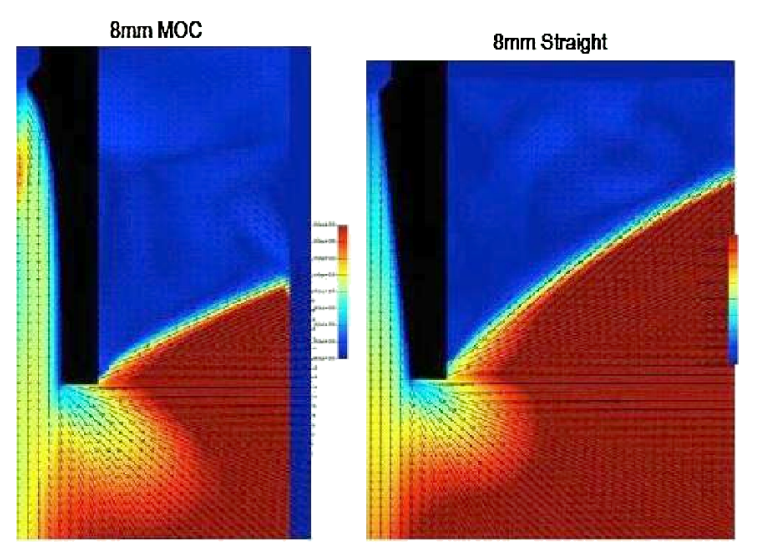

FIG. 10. Mach number contours for the $8 \mathrm{~mm} \mathrm{MOC}$ and $8 \mathrm{~mm}$ straight nozzles with velocity vectors superimposed on the flow fields.

with minimized scale length for the ramp up from vacuum at the edges. The former is required to keep the accelerator structure wavelength constant, while the latter is important for coupling of the laser into the jet [12]. The plasma densities required are in the range of $10^{18}-10^{19} / \mathrm{cc}$ which is accessible by any of these nozzles using a $<1000$ psi source pressure accessible with the present valve. Hence, the $4.6 \mathrm{~mm}$ straight nozzle is most desirable, as it shows the flattest profile across the jet. Second, the flow collimation near the nozzle exit is not significantly better for the $8 \mathrm{~mm}$ MOC nozzle than for the $8 \mathrm{~mm}$ straight nozzle. This is quite significant. If the machining costs of a straight nozzle are substantially less than that of a MOC nozzle, it would seem that the best choice, considering both flow field uniformity and intensity and cost effectiveness, would be to utilize the $4.6 \mathrm{~mm}$ straight nozzle over either the $8 \mathrm{~mm}$ straight nozzle or the $8 \mathrm{~mm}$ MOC nozzle. This nozzle shows the flattest profile across the jet.

\section{PARAMETRIC PERFORMANCE OF THE FAST VALVE}

Before measuring the gas flow profiles from the supersonic nozzles, we first performed a parametric study of the opening of the valve itself. For these measurements, the $4.6 \mathrm{~mm}$ straight nozzle was connected to the valve and an Endevco Model 8507C piezoresistive pressure transducer was used to measure the static pressure about $3 \mathrm{~mm}$ downstream of the nozzle exit and $\approx 1 \mathrm{~mm}$ below the center line.

Figure 11 shows the calculated valve motion normalized and superimposed upon the measured pressure when the plenum was charged to 800 psia helium. The measured pressure is also normalized to unity. Since helium moves at an average speed of $\approx 1250 \mathrm{~m} / \mathrm{s}$ between the throat and the nozzle exit, it takes $8 \mu \mathrm{s}$ for the gas to reach the location of the piezosensor. To account for this delay, the time base of the piezotrace has been shifted to the 


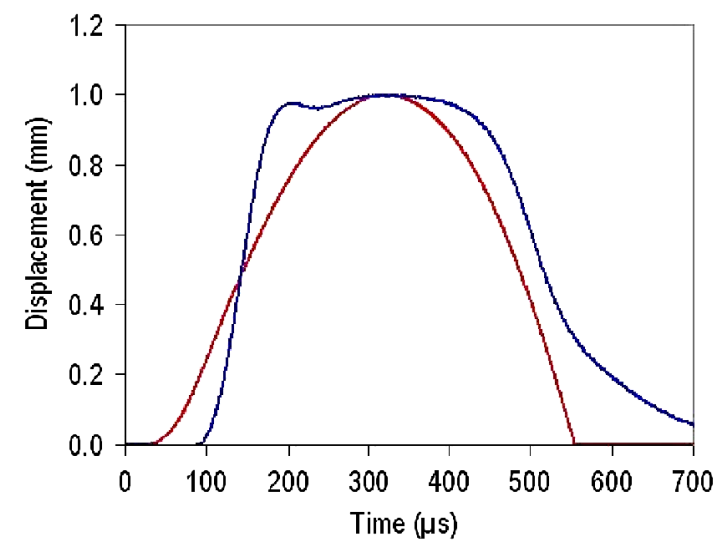

FIG. 11. Flyer-plate motion and measured piezopressure signal (normalized, $3 \mathrm{~mm}$ from nozzle exit: 800 psia helium, $350 \mathrm{~V}$ charge).

left by $8 \mu$ s. Figure 11 shows that the gas begins to flow at the valve seat $100 \mu \mathrm{s}$ after the current pulse begins, at which time the flyer plate has moved $20 \%$ (or $0.12 \mathrm{~mm}$ ) from its initial position. This means that the O-ring between the flyer-plate snout and the valve seat was initially compressed by $0.12 \mathrm{~mm}$. When the flyer plate has reached $70 \%$ of its full excursion $(0.4 \mathrm{~mm})$, the gas pressure reaches a maximum and remains flat thereafter. The area of the throat of the nozzle is $\approx 0.27 \mathrm{~mm}^{2}$. When the flyer plate has moved back $0.4 \mathrm{~mm}$, the annular area around the opening through which gas rushes inwards is $\approx 3.3 \mathrm{~mm}^{2}$. Hence, the flow is anticipated to reach Mach 1 at the nozzle throat as desired. The $\approx 80 \mu$ s delay for the pressure to reach the quasisteady state is due to the time required for the in-rushing gas to fill the "stilling" volume between the valve seat and nozzle throat. This stilling process occurs via a series of shocks and expansion waves that bounce back and forth between the annular opening in the flyer plate and the nozzle throat. For the typical distance between these two locations of $10 \mathrm{~mm}$, the round-trip time for sound waves is $\approx 20 \mu \mathrm{s}$; it takes a few such round-trip

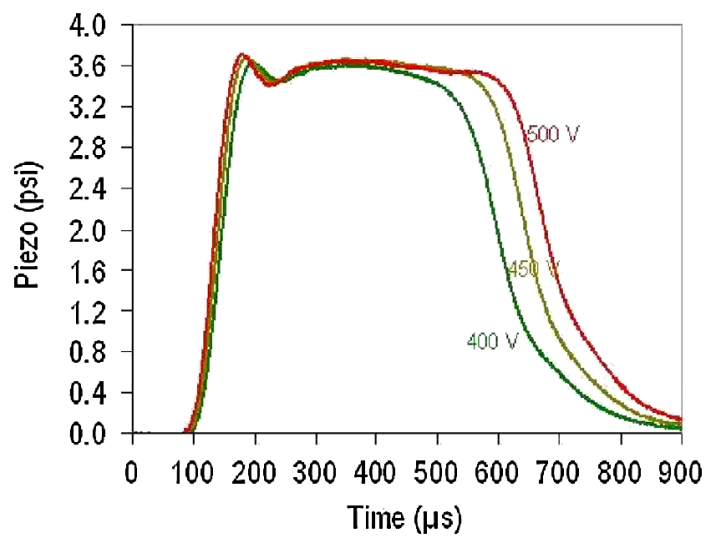

FIG. 12. Piezopressure (psi) vs time for 800 psia He at various capacitor charge voltages.

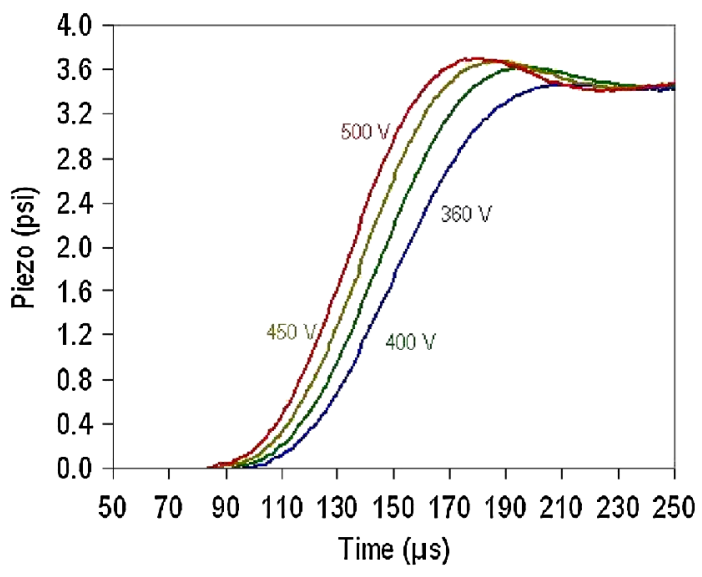

FIG. 13. Expanded view of Fig. 7 to show how the rise time of the gas is affected by charge voltage (coil current).

times to still the volume and create a quasisteady flow. The harmonic half period of the calculated flyer-plate/spring oscillator is $\approx 600 \mu$ s which is consistent with the observed closing time.

Figure 11 shows that, when the flyer plate returns to $<0.4 \mathrm{~mm}$, the flow begins to shut off, but the gas is still flowing after the valve has completely sealed against the O-ring. This is because the stilling volume is still filled with nearly 800 psia helium, which is subsequently evacuated by the nozzle outflow. It may be shown that the decay of pressure in a finite volume $V$ of monatomic gas through a throat area $A$ at speed $u$ follows the exponential law [13]:

$$
p(t)=p_{o} \exp (-t / \tau), \quad \text { where } \tau=-1.54 \mathrm{~V} / \mathrm{Au} .
$$

For the nozzle design shown in Fig. $1, \tau \approx 150 \mu$ s and such a slow decay after the valve has closed is clearly visible in Fig. 11.

In summary, the basic operation of the fast valve as a simple harmonic oscillator that is driven by a fast electromagnetic impulse, with an opening time $<100 \mu$ s and an open duration of $<600 \mu \mathrm{s}$, is demonstrated. For

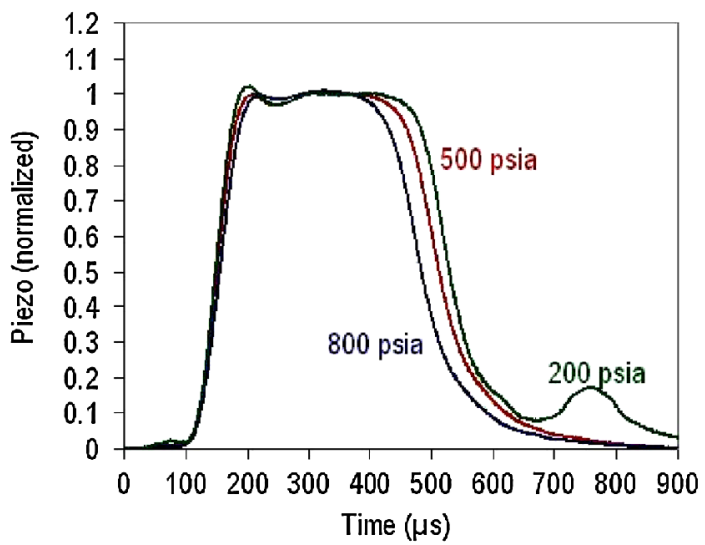

FIG. 14. Piezosensor signals at a fixed charge voltage of $350 \mathrm{~V}$ and different pressures. 


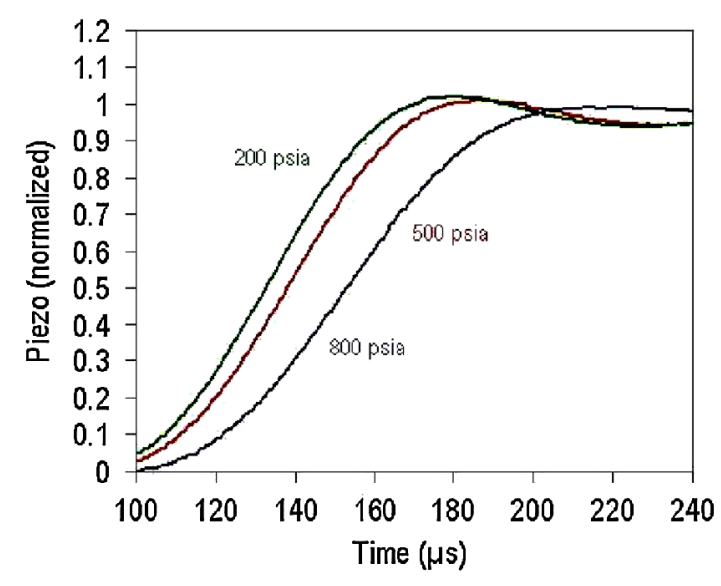

FIG. 15. Expanded view of Fig. 9 to show how rise time is affected by plenum pressure.

comparison, typical commercially available valves offer opening times that are a factor of 2-3 slower than this valve, when operating at $\sim 1000$ psia pressure. The closing times are substantially longer. We have compared the measured pressure vs time history in front of a supersonic nozzle with the calculated motion of the flyer plate and shown it to be as expected. These basic ideas may be used to extend the valve design to larger apertures or faster opening and closing times. Next we discuss the performance of the valve for various operational parameters

Figure 12 shows the piezosignal at different capacitor charge voltages, for a fixed plenum pressure of 800 psia. With increasing charge voltage (current to coil) the gas rises faster and lasts longer, as expected. The higher current gives greater acceleration to the flyer plate and, hence, increased travel resulting in a longer pulse width. Figure 13 shows an expanded view of these data, showing that the (10\%-90\%) rise time decreases from $65 \mu \mathrm{s}$ at $360 \mathrm{~V}$ to $50 \mu \mathrm{s}$ at $500 \mathrm{~V}$ charge.

Figure 14 shows data taken at a fixed charge voltage of $360 \mathrm{~V}$ but for varying pressures, from $200-800$ psia. As expected, the higher pressures close the valve faster, due to the greater restoring force on the flyer plate when it returns towards the seal. Figure 15 shows that the rise time is also

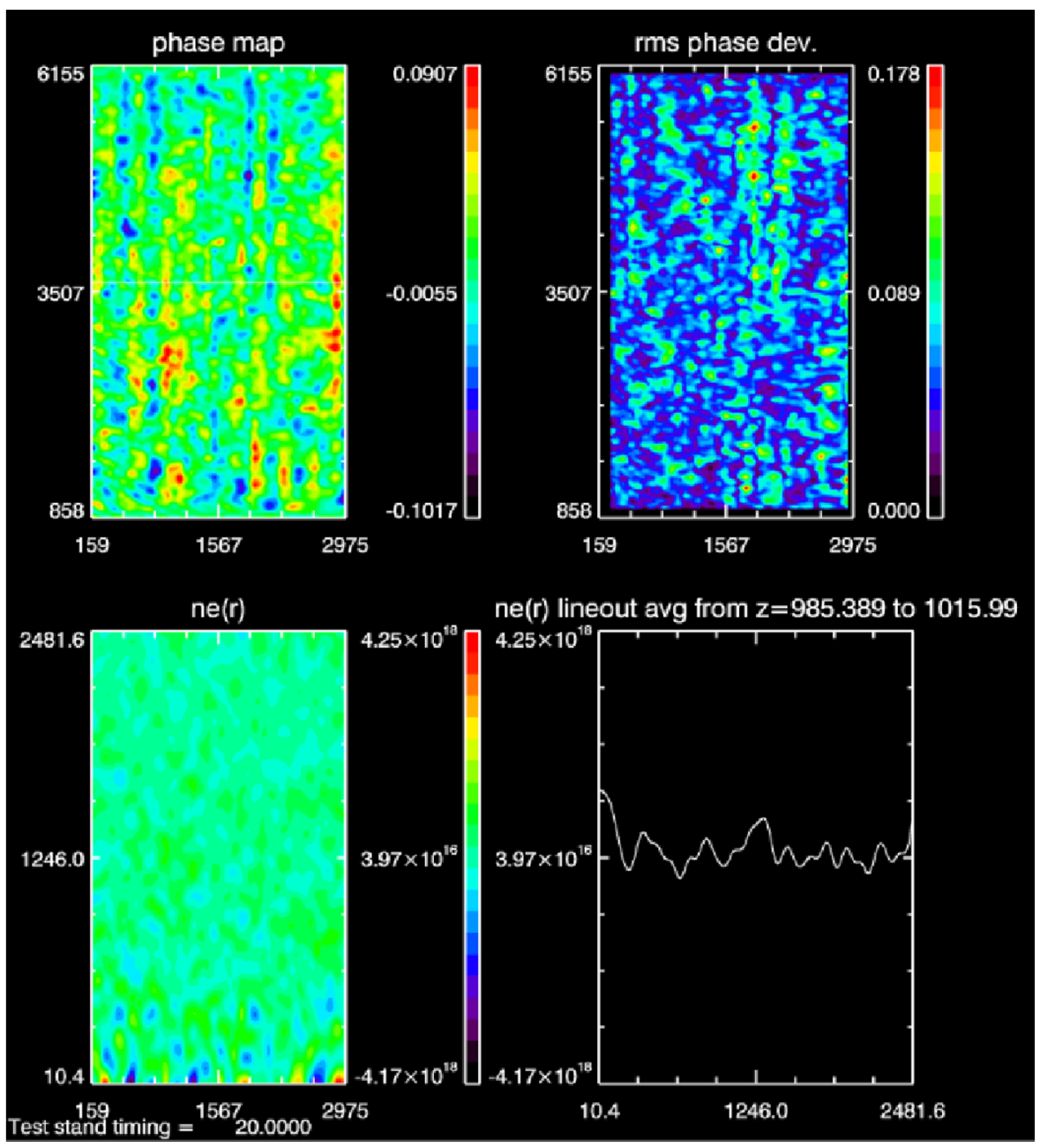

FIG. 16. Interferometer data from a He jet produced by the MOC nozzle at $615 \mathrm{psia} / 500 \mathrm{~V}$. Data snapshot at $20 \mu \mathrm{s}$ after valve is fired (no gas flow as yet). The top left is the phase map, the top right is the rms phase deviation; the bottom left is the derived (Abel inverted) density map and the bottom right is a "lineout" at about $1 \mathrm{~mm}$ from the nozzle face. 
faster at lower pressure, as expected. The late time bump in the data for 200 psia is due to a bounce of the flyer-plate stub when it returns to its seat. At higher pressures this bounce is eliminated.

This section has presented data that show that our fast valve is well suited to drive nozzles at pressures appropriate for LPAs, over length scales of $\sim$ few $\mathrm{mm}$ across the jets. By making the flyer-plate stub larger in diameter, it is straightforward to drive a nozzle with a larger throat and, hence, a larger exit aperture. In this way, a $\sim 1 \mathrm{~cm}$ gas jet may be produced with $\sim 10^{19} \mathrm{~cm}^{-3}$ gas density, suitable for $\sim 1 \mathrm{GeV}$ LPA tests.

The next section presents data on the gas flow obtained using an interferometer to demonstrate that suitable targets are produced for LPAs as an example application for the valve.

\section{INTERFEROMETER DATA}

A Mach-Zehnder interferometer at LOASIS/LBNL was used to map the density profiles at various times in the flow from two $8 \mathrm{~mm}$ nozzles, the MOC and the straight nozzle, to confirm the piezomeasurements of density and temporal flow profile, and to obtain data to compare with simulated density profiles. The interferometer used a continuous HeNe laser beam to probe the gas flow, and a CCD with a fast shutter to obtain images of the gas flow with $\sim 10 \mu \mathrm{s}$ time resolution. The gas plume imposed a phase delay on the laser beam proportional to the line integral of density, which was extracted by shearing techniques, and the density map was extracted by Abel inversion assuming axial symmetry of the flow. Details of the interferometer and its operation were published in [12].

Figures 16 and 17 show representative interferometer data from the MOC nozzle in $\mathrm{He}$ at $615 \mathrm{psia} / 500 \mathrm{~V}$. Figure 16 shows the background at $20 \mu$ s after the valve is fired, when there is no gas flow. The background shows a randomly distributed phase deviation of $0.1 \mathrm{rad} \mathrm{rms}$, giving a "floor" on the detectable density derived of $4 \times 10^{16} \mathrm{~cm}^{-3}$.

Figure 17 shows interferometer data at $220 \mu$ s, after the flow is fully developed. The phase map and derived density

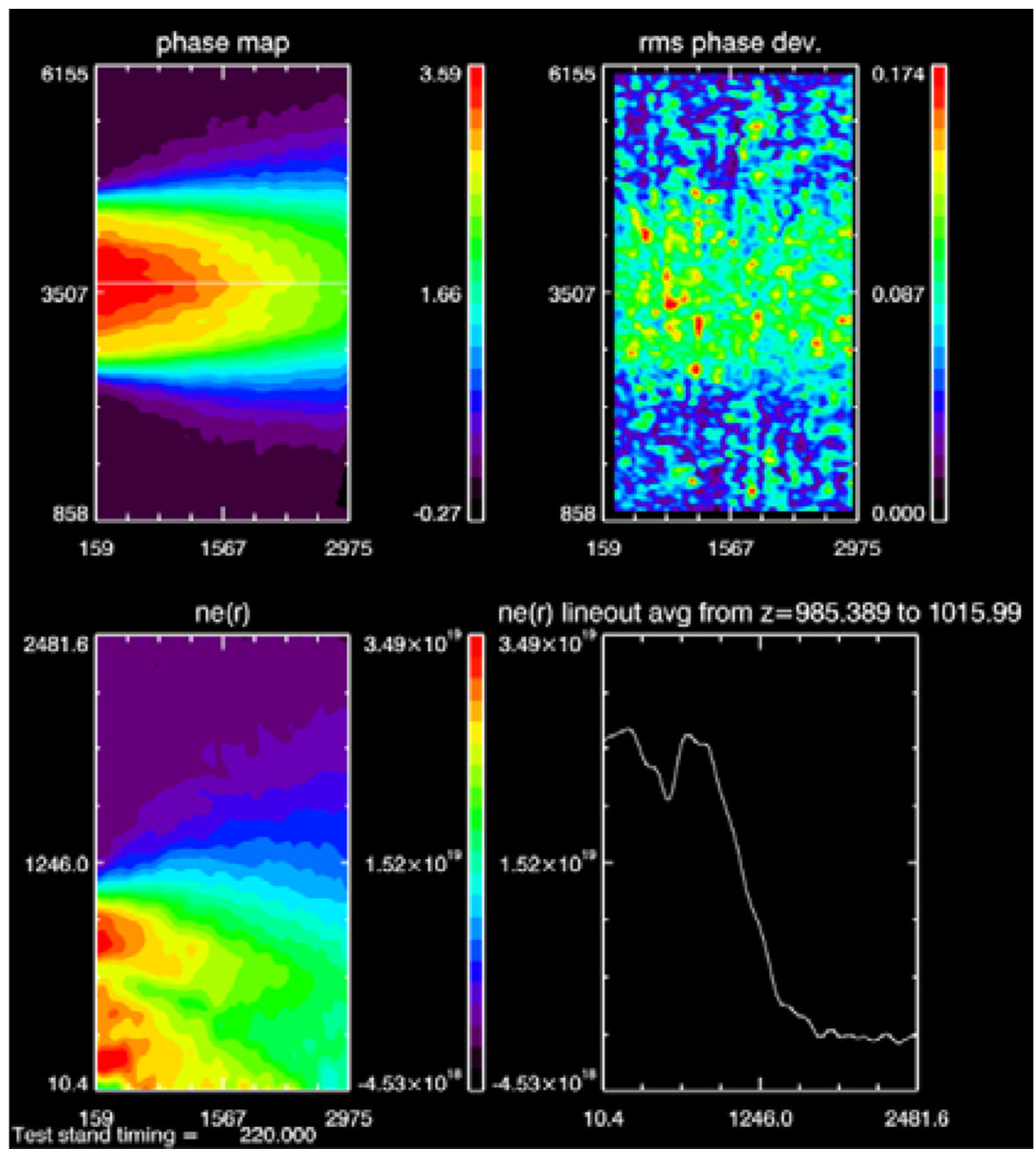

FIG. 17. Interferometer data from a He jet produced by the MOC nozzle at $615 \mathrm{psia} / 500 \mathrm{~V}$. Data snapshot at $220 \mu \mathrm{s}$ after valve is fired (gas flow is fully developed). 


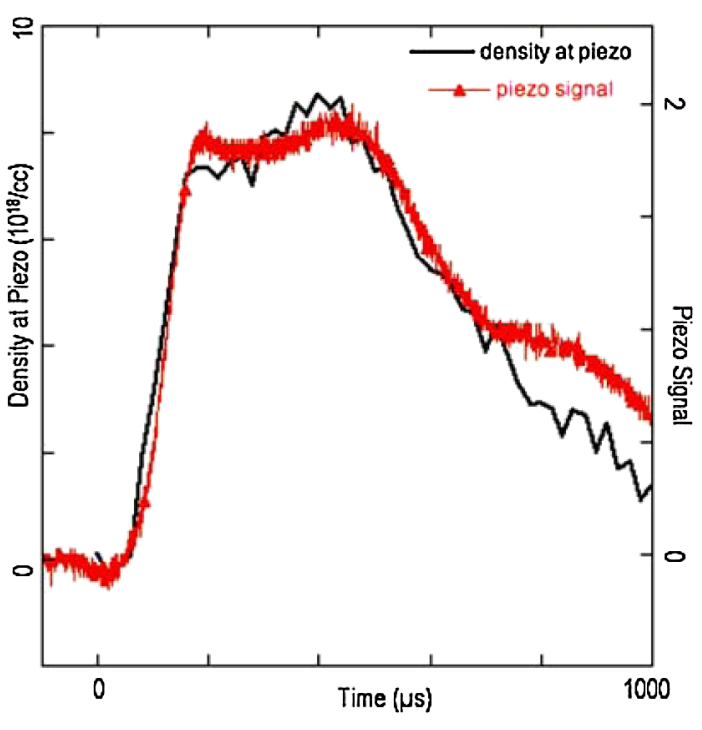

FIG. 18. Comparison of piezopressure vs time signal with density data derived from the laser interferometer.

map show a well collimated, high Mach number flow as expected for this nozzle. The maximum phase is $3.6 \mathrm{rad}$ with a $0.1 \mathrm{rms}$ deviation. The signal/noise is thus $\approx 36: 1$, corresponding to a $3 \%$ resolution. The actual rms variation from shot-shot is better than this value, which is a testament to the reproducibility of the valve. The piezodata also indicated $\mathrm{a} \leq \pm 2 \%$ shot-shot variation.

The radial lineout of the density shows a density of $\approx 2 \times 10^{19} \mathrm{~cm}^{-3}$, which is consistent with the Mach number and the plenum pressure that was used.

Figure 18 compares the measured pressure vs time data obtained using the piezoresistive transducer (located transverse to the flow about $2 \mathrm{~mm}$ downstream of the nozzle exit) with density data from the transverse laser interferometer. The data show relatively good agreement, indicating that the piezosensor data on flow time profile is accurate.

Figure 19 plots the measured pressure on the plateau of the flow vs plenum pressure. The linear fit to the peak

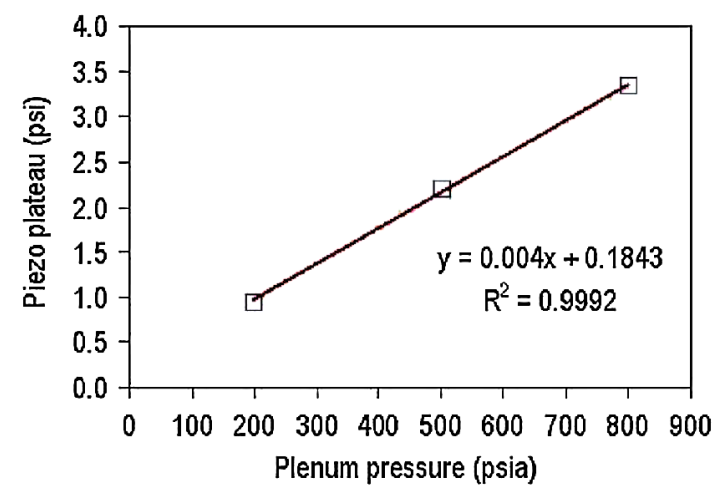

FIG. 19. Linearity of valve opening shown by local (static) pressure vs plenum pressure measured using piezosensor. pressure is excellent $\left(R^{2}=0.999^{2}\right)$ and gives a slope of $4 \times 10^{-3} \mathrm{psi} / \mathrm{psia}$. Assuming a Mach 5.7 expansion out of the nozzle, the ideal temperature in the expanded flow would be [13]

$$
\frac{T}{T_{o}}=\left[1+\frac{\gamma-1}{2} M^{2}\right]^{-1} \approx 25 \mathrm{~K}
$$

For an ideal gas, using this temperature the slope of the line in Fig. 11 may be converted into a number density coefficient, viz.

$$
\rho=7.7 \times 10^{16} \mathrm{~cm}^{-3} / \text { psia. }
$$

For example, Eq. (4) predicts that a 1000 psia plenum pressure would produce a nozzle flow with atomic number density of $\approx 7.7 \times 10^{19} \mathrm{~cm}^{-3}$ about $2 \mathrm{~mm}$ from the nozzle exit. The interferometer data gave an inferred electron density of $8 \times 10^{19} \mathrm{~cm}^{-3}$ at $1 \mathrm{~mm}$ from the nozzle at $1000 \mathrm{psig}$. This would correspond to a $\mathrm{He}$ atom density of $4 \times 10^{19} \mathrm{~cm}^{-3}$, which is half the value obtained from the piezodata. Similarly the isentropic flow equations [13] would predict 2 psi in the flow (for 1000 psia in the plenum) whereas the line in Fig. 11 indicates 4 psi. If the piezosensor were not exactly on the flow center line, there could well have been some stagnation on the face, which could easily account for a slightly higher pressure than the true value. To quantify this possible error, suppose that the face of the sensor is not parallel to the flow but at an angle $\theta$ to it. The actual pressure measured $p_{m}$ would be [13]

$$
p_{m}=p_{\text {true }}\left(1+\gamma M^{2} \sin \theta\right) .
$$

A misalignment of just 2.2 deg would suffice to give a 2 times higher apparent pressure than the true static pressure of the $M=5.7$ flow.

\section{OPERATION AT HIGH REPETITION RATES}

The circuit equation for the driver and the measured resistance of the spiral coil indicate that $\approx 10 \mathrm{~J} /$ pulse is dissipated in the coil. The spiral coil was pressed into a copper bushing which was itself press fit into the stainless steel valve housing. This bushing served as a good heat conductor to transfer the heat from the spiral coil to the larger thermal mass of the valve body. The valve was fired repetitively at $0.5 \mathrm{~Hz}$ for an hour at a time and required no cooling. The surface temperature of the valve body was measured by a thermocouple to rise to $39^{\circ} \mathrm{C}$ after two hours of continual (repetitive) operation. Operation at $>1 \mathrm{~Hz}$ would require active cooling of the housing. This may be accomplished by replacing the stainless steel housing with a copper housing and welding a water flow jacket to this housing, as the copper bushing would efficiently transport the heat out of the coil and into the 
cooling jacket. The present valve was fired at up to $10 \mathrm{~Hz}$ rep-rates in short bursts, without cooling. The rep-rate was not limited by heating but rather by the fact that the silicon controlled rectifier (SCR) switch in the driver circuit did not turn off completely between pulses and eventually stayed clamped on, forcing the valve to be turned off. By replacing the SCR switch with an insulated gate bipolar transistor (which may be actively turned off between pulses) and implementing cooling, we expect to be able to operate at $10 \mathrm{~Hz}$ or higher in the future.

\section{CONCLUSIONS}

A design has been presented for a fast electromagnetic valve that could be advantageous for several applications such as fast, high current $z$ pinches, laserplasma accelerators. and for membrane-free, fast pulsed, metered gas injection in conventional accelerators. The prototype valve opens in $<100 \mu \mathrm{s}$ and closes in $<500 \mu \mathrm{s}$, at pressures of up to 800 psia with rep-rates of $1 \mathrm{~Hz}$ (up to $10 \mathrm{~Hz}$ with cooling). The design features allow extending the operation to $\sim 1500$ psia pressures as the driving force on the coil greatly exceeds the restoring forces due to the gas pressure and the spring. The three key physical parameters that govern the opening and closing are the electromagnetic force, the flyerplate mass, and the spring constant. Further increasing the force while increasing its frequency would allow a smaller flyer-plate mass and allow still faster opening, in $\sim 20 \mu \mathrm{s}$ or less. In turn, using a higher spring constant would allow faster closing, in $<250 \mu$ s.

\section{ACKNOWLEDGMENTS}

This research was supported by the U.S. Department of Energy SBIR Grant No. DE-FG02-08ER85030, by High Energy Physics grants including No. DE-AC0205CH11231, and by NA-22.

[1] C. G. R. Geddes, C. Toth, J. van Tilborg, E. Esarey, C. B. Schroeder, D. Bruhwiler, C. Nieter, J. Cary, and W. P. Leemans, Nature (London) 431, 538 (2004).

[2] S. P. D. Mangles et al., Nature (London) 431, 535 (2004).

[3] J. Faure et al., Nature (London) 431, 541 (2004).

[4] W. P. Leemans, B. Nagler, A. J. Gonsalves, C. Toth, K. Nakamura, C. G. R. Geddes, E. Esarey, C. B. Schroeder, and S. M. Hooker, Nature Phys. 2, 696 (2006).

[5] C. G. R. Geddes et al., Phys. Rev. Lett. 100, 215004 (2008).

[6] A. J. Gonsalves et al., Phys. Rev. Lett. 98, 025002 (2007).

[7] W. R. Gentry and C. F. Giese, Rev. Sci. Instrum. 49, 595 (1978).

[8] E. J. Corat and V. J. Trava-Airoldi, Rev. Sci. Instrum. 61, 1068 (1990).

[9] T.E. Adams, B. H. Rockney, R. J. S. Morrison, and E. R. Grant, Rev. Sci. Instrum. 52, 1469 (1981).

[10] M. Krishnan, J. Wright, and T. Ma, Proceedings of the 13th Advanced Accelerator Concepts Workshop, Santa Cruz, CA, AIP Conf. Proc. No. 1086 (AIP, New York, 2008).

[11] S. Semushin and V. Malka, Rev. Sci. Instrum. 72, 2961 (2001).

[12] C.G.R. Geddes, Ph.D. dissertation, University of California Berkeley, 2005.

[13] H.W. Liepmann and A. Roshko, Elements of Gas Dynamics (Dover Publications, New York, 2002). 